\title{
First-principles study of the lattice dynamics ofK2SO4
}

\section{Citation}

Liu, D., H. M. Lu, F. G. Ullman, and J. R. Hardy. 1991. "First-Principles Study of the Lattice Dynamics ofK2S04." Physical Review B 43 (7) (March 1): 6202-6205. doi:10.1103/ physrevb.43.6202.

\section{Published Version}

doi:10.1103/PhysRevB.43.6202

\section{Permanent link}

http://nrs.harvard.edu/urn-3:HUL.InstRepos:32418715

\section{Terms of Use}

This article was downloaded from Harvard University's DASH repository, and is made available under the terms and conditions applicable to Other Posted Material, as set forth at http:// nrs.harvard.edu/urn-3:HUL.InstRepos:dash.current.terms-of-use\#LAA

\section{Share Your Story}

The Harvard community has made this article openly available.

Please share how this access benefits you. Submit a story.

Accessibility 


\title{
First-principles study of the lattice dynamics of $\mathrm{K}_{2} \mathrm{SO}_{4}$
}

\author{
D. Liu, H. M. Lu, F. G. Ullman, and J. R. Hardy \\ Department of Physics and Center for Electro-Optics, University of Nebraska-Lincoln, Lincoln, Nebraska 68588-0111
}

(Received 20 July 1990)

\begin{abstract}
Using a newly developed first-principles approach to simulations of ionic molecular crystals, we performed static relaxation, molecular-dynamics simulation, and lattice-dynamics calculations, and measurements of the Raman spectrum, for the Pnam structure of $\mathrm{K}_{2} \mathrm{SO}_{4}$. It was found that the structure does not have the zone-center instability present in isomorphous $\mathrm{K}_{2} \mathrm{SeO}_{4}$ found in an earlier study. This difference between the two systems is attributed to the different charge distributions in the molecular ions. The calculated Raman-active zone-center frequencies for the Pnam structure of $\mathrm{K}_{2} \mathrm{SO}_{4}$ were found to be in general agreement with the experimental Raman frequencies.
\end{abstract}

First-principles simulations using $a b$ initio Gordon$\mathrm{Kim}^{1}$ pair potentials have proven very successful in the studies of phase transitions, lattice dynamics, and other important properties of complex ionic solids. ${ }^{2-8}$ Based on the rigid-free-ion electron charge densities and the electron-gas model, these pair potentials give accurate descriptions of the potential-energy surfaces for ionic crystals. Recently this approach has been extended to treat molecular ionic solids, i.e., solids that contain molecular ions, e.g., $\mathrm{SO}_{4}{ }^{2-}, \mathrm{SeO}_{4}^{2-}, \mathrm{NO}_{2}{ }^{-}$, etc., where the dominant bonding within the molecular ions is not ionic, but covalent. 9,10 By starting from $a b$ initio quantum-chemistry calculations for the whole molecular ion, and then using the resultant electron charge density to calculate pair potentials, the approach accounts properly for the effects of intramolecular covalency on the intermolecular interactions. For the intramolecular interactions, which are beyond the Gordon-Kim model and the pairwise interaction scheme, a harmonic expansion is employed, with the expansion coefficients also determined by $a b$ initio quantum-chemistry calculations. When applied to study the phase transitions in $\mathrm{K}_{2} \mathrm{SeO}_{4}$, an important member of the family of $A_{2} B X_{4}$ compounds, the method successfully reproduced, via supercell molecular-dynamics simulations, the transition from the room-temperature paraelectric phase to the lowertemperature ferroelectric superstructure. Also, firstprinciples lattice-dynamics calculations for the static room-temperature structure clearly revealed the structural instabilities in the system which were in excellent agreement with the experimentally observed soft-phonon behavior of the incommensurate phase transition.

In the present work we apply this method to the study of $\mathrm{K}_{2} \mathrm{SO}_{4}$, another member in the $A_{2} B X_{4}$ family. At room temperature, $\mathrm{K}_{2} \mathrm{SO}_{4}$ is isomorphous with $\mathrm{K}_{2} \mathrm{SeO}_{4}$ and has the same orthorhombic to hexagonal phase transition at high temperatures. ${ }^{11,12}$ However, it does not have any known incommensurate phase transition at lower temperatures. The only transition below room temperature was found to be at $56 \mathrm{~K}$, and showed no obvious ferroelectric properties. ${ }^{13}$ Thus this low temperature phase transition in $\mathrm{K}_{2} \mathrm{SO}_{4}$ is definitely different from that for $\mathrm{K}_{2} \mathrm{SeO}_{4}$. It is thus important to see if our $a b$ initio theoretical studies can account for the similarities and differences between the two chemically similar systems and reveal the origin of these differences at the level of interionic interactions.

One of the most interesting findings in the previous study ${ }^{10}$ of $\mathrm{K}_{2} \mathrm{SeO}_{4}$ is that the room-temperature Pnam $\left(D_{2 h}^{16}\right)$ structure has not only the instability that drives the incommensurate and ferroelectric cell-tripling transition seen experimentally, but also a zone-center instability. This is a direct reflection of the double-well structure of the potential-energy surface responsible for the observed phase transitions. Therefore, in the present study, we put our emphasis on the lattice dynamics of $\mathrm{K}_{2} \mathrm{SO}_{4}$ at the zone center. Also we compare our first-principles normal-mode frequencies with the measured Raman frequencies; such a comparison will provide a valuable test of the theoretical description of the system.

Raman scattering measurements on $\mathrm{K}_{2} \mathrm{SO}_{4}$ have been reported by a number of workers. ${ }^{14-16}$ At room temperature, the spectral resolution is rather poor and there is disagreement among these results. In order to achieve higher resolution for better comparison with the theoretical frequencies, we performed Raman scattering measurements at liquid-nitrogen temperature. The experimental details will be given elsewhere. ${ }^{17}$ The measured Raman frequencies are listed in Table II. These frequencies agree well with a previous Raman measurement at the same temperature. ${ }^{16}$

Our theoretical study started from $a b$ initio quantumchemistry calculations for the whole $\mathrm{SO}_{4}{ }^{2-}$ ion. These calculations did not include a background crystal field. This is justified by the finding that, while the oxygen sites are the only sites with non-negligible crystal fields in the Pnam structure of $\mathrm{K}_{2} \mathrm{SO}_{4}$, over $90 \%$ of the contribution to these fields is from the other ions in the same $\mathrm{SO}_{4}{ }^{2-}$ group, rather than from the rest of the lattice. Although the background crystal field may affect, to some degree, the shapes of the tails of the electron charge distributions on the outskirts of the $\mathrm{SO}_{4}{ }^{2-}$ ion, it is relatively unimportant in determining the electron charge distribution within the $\mathrm{SO}_{4}{ }^{2-}$ ion which is most crucial for both intra- 
and intermolecular interactions. A full description of the procedure for obtaining interionic potentials from these quantum-chemistry calculations has been given in Ref. 10. Briefly, we first performed a structural optimization for a $\mathrm{SO}_{4}^{2-}$ ion; the optimized structure is a perfect tetrahedron with the $\mathrm{S}-\mathrm{O}$ bond length of 2.81 a.u., which is fairly close to the experimental value of 2.72 a.u. at room temperature. Then the second derivatives of the energy with respect to distortions of the $\mathrm{SO}_{4}{ }^{2-}$ ion are calculated to construct a harmonic expansion for the description of the intramolecular interactions. The resultant electron charge density for the $\mathrm{SO}_{4}{ }^{2-}$ ion is decomposed into approximate charge densities for the individual ions. ${ }^{10}$ Using these charge densities and the free-ion charge density for the $\mathrm{K}^{+}$ion, ${ }^{18}$ we then computed the short-range pair potentials between these ions according to the Gordon-Kim ${ }^{1}$ electron-gas model. The charges on the ions were +1 for $K,+1.6372$ for $S$, and -0.9093 for $\mathrm{O}$, where the fractional charges were obtained from the computed charge density of $\mathrm{SO}_{4}{ }^{2-}$ by a Mulliken population analysis. ${ }^{19}$

With the intra- and intermolecular potentials determined, we first performed static relaxation for the roomtemperature Pnam structure of $\mathrm{K}_{2} \mathrm{SO}_{4}$. Our relaxation started from the experimental structure and was subject to the constraints of Pnam symmetry, i.e., only the structural parameters listed in Table I were allowed to vary. The energy minimization was for an infinite lattice, obtained by applying periodic boundary conditions, and followed a Newton-Raphson algorithm. The standard technique of the Ewald sum was used for the calculation of the lattice energy and forces, etc.

The relaxation quickly reaches the structure with zero forces on the basis ions and zero stresses. The parameters for the relaxed structure are given in Table I, along with the experimental values. As can be seen, all the lattice constants in the theoretical structure are shorter than the experimental values, ${ }^{11} 5 \%$ for $a, 4 \%$ for $b$, and $3 \%$

TABLE I. Experimental (Ref. 11) and theoretical structural parameters for the Pnam structure of $\mathrm{K}_{2} \mathrm{SO}_{4}$. (The lattice constants are given in angstroms.)

\begin{tabular}{ccl}
\hline \hline Parameters & Experiment & Theory \\
\hline$a$ & 7.497 & 7.097 \\
$b$ & 10.083 & 9.667 \\
$c$ & 5.735 & 5.581 \\
$x / a$ of $S(1)$ & 0.2315 & 0.2330 \\
$y / b$ of $S(1)$ & 0.4208 & 0.4195 \\
$x / a$ of $K(1,1)$ & 0.1755 & 0.1683 \\
$y / b$ of $K(1,1)$ & 0.0884 & 0.0882 \\
$x / a$ of $K(2,1)$ & 0.9906 & 0.9897 \\
$y / b$ of $K(2,1)$ & 0.7052 & 0.7008 \\
$x / a$ of $O(2,1)$ & 0.2899 & 0.3100 \\
$y / b$ of $O(2,1)$ & 0.5576 & 0.5613 \\
$x / a$ of $O(3,1)$ & 0.0454 & 0.0258 \\
$y / b$ of $O(3,1)$ & 0.4157 & 0.4229 \\
$x / a$ of $O(1,1)$ & 0.2996 & 0.3010 \\
$y / b$ of $O(1,1)$ & 0.3530 & 0.3468 \\
$z / c$ of $O(1,1)$ & 0.0426 & 0.0348 \\
\hline \hline
\end{tabular}

for $c$, which is a rather general feature for large unit cell simulations using Gordon-Kim potentials. Although these deviations are certainly not negligible in magnitude, the fact that they are percentagewise comparable in all three directions indicates that the theoretical structure is almost uniformly contracted and thus still preserves the basic physical character of the system.

The theoretical basis parameters are very close to the experimental values. The largest difference is 0.02 for $x / a$ of $O(2,1)$, which gives a displacement of about 0.15 $\AA$, well below the thermal fluctuations in the positions of these ions at room temperature.

In the previous study ${ }^{10}$ of $\mathrm{K}_{2} \mathrm{SeO}_{4}$ a similar Pnamconstrained static relaxation was performed and equally good agreement with the experimental structure was found, which seemed to indicate the stability of the Pnam structure. However, when a molecular-dynamics relaxation was performed for a single Pnam unit cell (without the symmetry constraints), the system transformed into a monoclinic structure, which directly revealed the zonecenter instability in the room-temperature phase. We therefore performed a molecular-dynamics relaxation for a Pnam unit cell of $\mathrm{K}_{2} \mathrm{SO}_{4}$. Our relaxation follows a constant (zero) -pressure algorithm ${ }^{20}$ with the time step size of 0.005 ps. Starting from the experimental roomtemperature structure, we gradually reduced the kinetic energy of the sample and thus reached $T=0 \mathrm{~K}$. We found that the atomic positions and the potential energy of the relaxed structure are almost identical with those for the theoretical Pnam structure given in Table I. Consequently, there is no structural transformation and therefore no zone-center instabilities, as will also be apparent from subsequent lattice-dynamical calculations.

Next we computed the normal-mode frequencies at the zone center for the theoretical Pnam structure of $\mathrm{K}_{2} \mathrm{SO}_{4}$. The resultant normal modes are classified according to symmetry and the Raman-active frequencies are listed in Table II, along with our measured Raman frequencies at liquid-nitrogen temperature and the percentage differences $\delta=\left[\left(\omega_{t}-\omega_{e}\right) / \omega_{e}\right] \times 100 \%$, with the $\omega_{t}$ and $\omega_{e}$ being the theoretical and experimental frequencies, respectively. No imaginary frequencies were found, and therefore there is no zone-center instability in the system, consistent with the molecular-dynamics result. This constitutes a major difference between $\mathrm{K}_{2} \mathrm{SO}_{4}$ and $\mathrm{K}_{2} \mathrm{SeO}_{4}$.

The agreement between the theoretical and experimental frequencies in Table II is generally acceptable, considering the parameter-free nature of the potentials. In terms of the absolute values, the lattice modes seem to agree better than the internal modes that originate from the internal vibrations of the $\mathrm{SO}_{4}{ }^{2-}$ molecular ions. But the percentage differences are comparable, and for almost all modes are within $\pm 20 \%$, with only a few exceptions. It should be pointed out that since the present calculation was for a statically fully relaxed structure, it did not include the effects of temperature and therefore direct comparison with experiment can be misleading. We found that if we increase the lattice constants in the theoretical structure by about $0.1 \%$ to their values at $80 \mathrm{~K}$ (obtained by a molecular-dynamics simulation) to include temperature effects, the fit between theory and experiment clearly 
TABLE II. Experimental (four of the $B_{1 g}$ modes were not observed due to their weak intensities) and calculated Raman frequencies (in $\mathrm{cm}^{-1}$ ) for Pnam $\mathrm{K}_{2} \mathrm{SO}_{4}$.

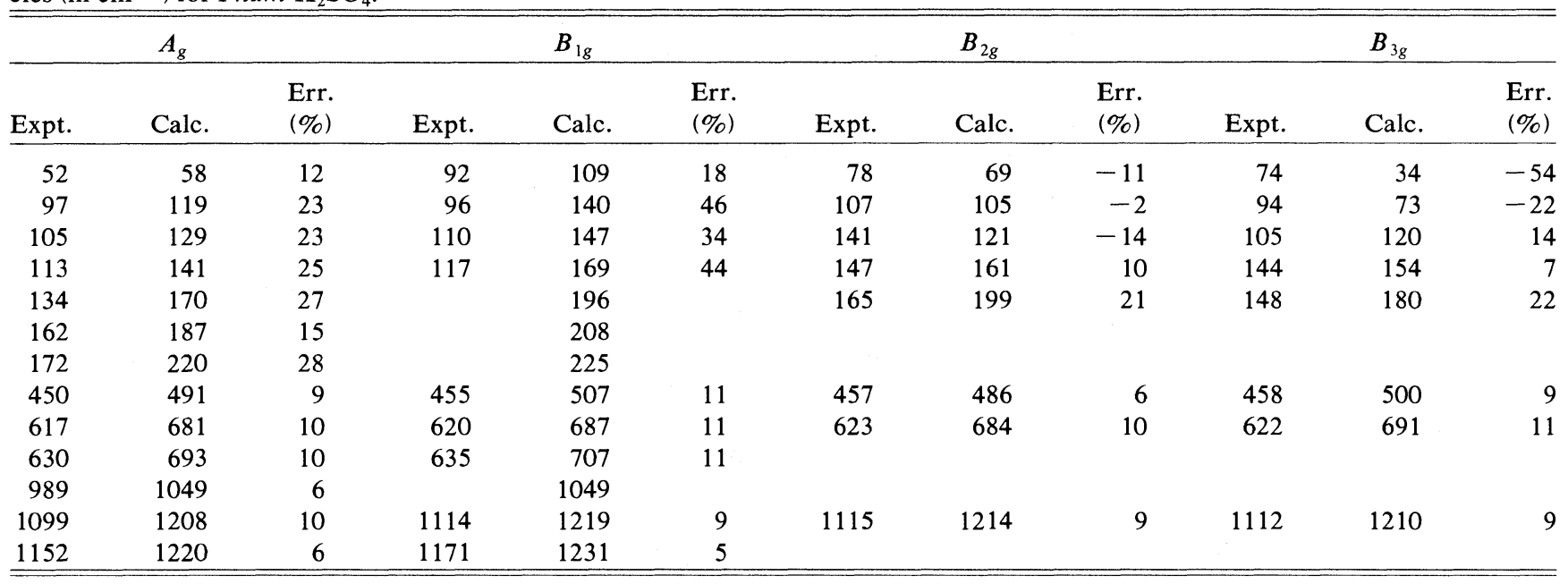

improves.

Furthermore, it would be unwise to infer from these discrepancies, that the potential-energy surface has only comparable accuracy, since there is good reason to believe that such differences are to be expected. This stems from the fact that oxygen ions are very light. As a consequence, when moving at low frequencies, in a shallow well, they travel relatively very large distances and the motion is highly anharmonic. Consequently the curvature at the bottom of the well, which corresponds to our theoretical frequencies, may give only a semiquantitative agreement with the observed Raman frequency. As an example, the worst discrepancy, between 74 (observed) and $34 \mathrm{~cm}^{-1}$ (theory) for the lowest $B_{3 g}$ mode, is almost certainly explicable in these terms. At the other extreme, as has been noted, the very-high-frequency internal modes display (in absolute terms) even worse discrepancies. However, this is well known in quantum chemistry, and is again due to anharmonicity owing to the light oxygens having large zero-point amplitudes which (a) displace their mean positions from the potential well minima, and (b) soften the effective force constant to reflect the average curvature of the potential well at the ground-state level. In addition, these factors will also indirectly influence the low-frequency motions.

The zone-center instability found in $\mathrm{K}_{2} \mathrm{SeO}_{4}$ was due to the rotation of the $\mathrm{SeO}_{4}{ }^{2-}$ ions as rigid bodies from their orientations in the room-temperature structure. ${ }^{10}$ Thus we compared the intermolecular potentials in $\mathrm{K}_{2} \mathrm{SO}_{4}$ with those in $\mathrm{K}_{2} \mathrm{SeO}_{4}$ for an explanation for the absence of such zone-center instability in $\mathrm{K}_{2} \mathrm{SO}_{4}$. It was found that the short-range potentials in the two compounds are essentially the same in their effective ranges and that the only differences are the effective ionicities for the atoms within the molecular ions. In $\mathrm{K}_{2} \mathrm{SeO}_{4}$, they are 1.1504 for $\mathrm{Se}$ and -0.7876 for $\mathrm{O},{ }^{10}$ while in $\mathrm{K}_{2} \mathrm{SO}_{4}$ they are 1.6370 for $\mathrm{S}$ and -0.9093 for $\mathrm{O}$. A close inspection of Pnam structures of these compounds shows that of all the intermolecular ion pairs, the K-O interactions are the most important, since they are the shortest and are directly responsible for the rotation of the molecular ions. Therefore the difference between the lattice-dynamics properties in $\mathrm{K}_{2} \mathrm{SeO}_{4}$ and in $\mathrm{K}_{2} \mathrm{SO}_{4}$ can be mainly attributed to the difference in the ionicities of the oxygen atoms, which originates from the different chemistry between $\mathrm{SeO}_{4}{ }^{2-}$ and $\mathrm{SO}_{4}{ }^{2-}$ ions.

In summary, we have performed static relaxation, molecular-dynamics, and lattice-dynamics calculations for the room-temperature structure of the $\mathrm{K}_{2} \mathrm{SO}_{4}$ crystal. Unlike the isomorphous $\mathrm{K}_{2} \mathrm{SeO}_{4}$, no zone-center instability has been found. This implies that any soft-phonon type of phase transition below room temperature has to be associated with a non-zone-center instability and the resultant structure should be a superlattice.

This work was supported by the U.S. Army Research Office.
${ }^{1}$ R. G. Gordon and Y. S. Kim, J. Chem. Phys. 56, 3122 (1971).

${ }^{2}$ L. L. Boyer, Phys. Rev. Lett. 42, 584 (1979). A fuller account is given in Phys. Rev. B 23, 3673 (1981).

${ }^{3}$ L. L. Boyer, Phys. Rev. Lett. 45, 1858 (1980); 46, 1172 (1981).

${ }^{4}$ L. L. Boyer and J. R. Hardy, Phys. Rev. B 24, 2527 (1981).

${ }^{5}$ L. L. Boyer, J. Phys. C 17, 1825 (1984).

${ }^{6}$ V. Katkanant, P. J. Edwardson, J. R. Hardy, and L. L. Boyer,
Phys. Rev. Lett. 57, 2033 (1986).

${ }^{7}$ P. J. Edwardson, V. Katkanant, J. R. Hardy, and L. L. Boyer, Phys. Rev. B 35, 8470 (1987).

${ }^{8}$ V. Katkanant, P. J. Edwardson, and J. R. Hardy, Phase Transitions 15, 103 (1989).

${ }^{9}$ H. M. Lu and J. R. Hardy, Phys. Rev. Lett. 64, 661 (1990).

${ }^{10}$ H. M. Lu and J. R. Hardy, Phys. Rev. B 42, 8339 (1990). 
${ }^{11}$ J. A. Mcginnety, Acta Crystallogr. B 28, 2845 (1972).

${ }^{12}$ S. Shiozaki, A. Sawada, Y. Ishibashi, and Y. Takagi, J. Phys. Soc. Jpn. 43, 1314 (1977).

${ }^{13}$ K. Gesi, Y. Tominaga, and H. Urabe, Ferroelectrics Lett. 44, 71 (1982).

${ }^{14}$ M. Debeau, Rev. Phys. Appl. 7, 49 (1972).

${ }^{15}$ M. Scrocco, Phys. Status Solidi B 91, K21 (1979).

${ }^{16} \mathrm{~S}$. Montero and R. Schmolz, J. Raman Spectrosc. 2, 101
(1973).

${ }^{17}$ D. Liu, H. M. Lu, F. G. Ullman, and J. R. Hardy (unpublished).

${ }^{18}$ E. Clementi and C. Roetti, At. Data Nucl. Data Tables 14, 177 (1974).

${ }^{19}$ R. S. Mulliken, J. Chem. Phys. 23, 1833 (1955).

${ }^{20}$ M. Parrinello and A. Rahman, Phys. Rev. Lett. 45, 1196 (1980). 